\title{
The Detection of the Liquid Drop Fingerprint's Abnormal Values Based on Boxplot Method
}

\author{
Q. Song \\ Automation School \\ Beijing University of Posts and Telecommunications (BUPT) \\ Beijing, China \\ M.Y. Qiao \\ Automation School \\ Beijing University of Posts and Telecommunications (BUPT) \\ Beijing, China
}

\begin{abstract}
In order to effectively detect the abnormal data of the liquid drop in the droplet analysis system and to improve the accuracy of the liquid drop fingerprint, a new method based on boxplot is put forward. After optimizing the 12 dimensional feature vectors of the liquid drop fingerprint, visualization of statistics is applied on the optimized 6 dimensional feature vectors by using boxplot method. With the median $( \pm 5 \%)$ as the threshold values, abnormal droplets are screened. Experimental results show that the detection recognition ratio of the abnormal liquid drop can be ensured after feature optimization, together with the greatly reduced computational complexity. Boxplot method is effective in detection of abnormal liquid drop fingerprint, with its accuracy up to $100 \%$ among selected samples.
\end{abstract}

Keywords-the liquid drop fingerprint; abnormal values detectio; identification; Boxplot method

\section{INTRODUCTION}

The fiber-capacitive drop analyzing technology has been developing rapidly in recent years and its core is the liquid drop fingerprint. The liquid drop fingerprints are curve images obtained after filtering and normalizing, representing liquid characteristics by collecting the

Optical fiber capacitance data in the process of droplet formation by the fiber-capacitive drop analyzing technology. By identifying the liquid drop fingerprint, the type of liquid can be identified and the characteristic of liquid can be analysed [1].

The liquid drop fingerprint can be obtained by the drop analyzing technology, which reveals the variation regularity during the light signals pass through the liquid drop. The results of experiment showed that the liquid drop fingerprint reflects physical characteristics and chemical characteristics of droplet with accuracy and uniqueness under the certain conditions.

Effects of disturbance on the droplet analysis system first appeared on the original data, which will produce abnormal drop data. If processing is wrong with these abnormal data, it will make the liquid drop fingerprint abnormal, causing

\author{
S.H. Zhang \\ Automation School \\ Beijing University of Posts and Telecommunications (BUPT) \\ Beijing, China \\ L. Yang \\ Automation School \\ Beijing University of Posts and Telecommunications (BUPT) \\ Beijing, China
}

instability in the system. By writing abnormal droplet test procedure, disturbance effect on droplet fingerprint can be effectively removed.

\section{WAVEFORM ANALYSIS METHOD OF THE LIQUID DROP FINGERPRINT}

The liquid drop fingerprint is an important criterion to identify liquid, which contains the optical fiber voltage signal and the capacitor voltage signal in the process of droplet growth $[1,2]$. Removed the time series by normalizing, we can get the only Fingerprint Drop, which are shown in figure 1.

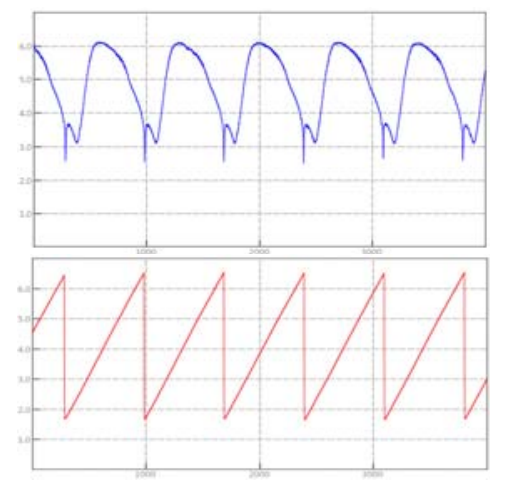

FIGURE I. THE FIBER VOLTAGE SIGNALS AND THE CAPACITOR VOLTAGE SIGNALS OF LIQUID DROPLET.

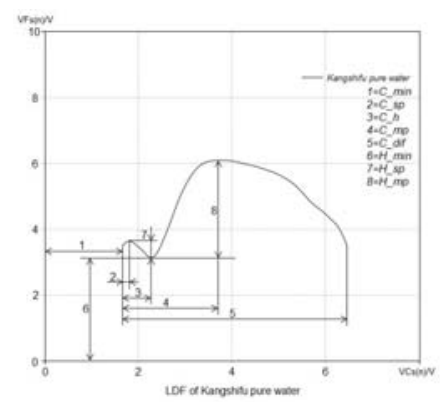

FIGURE II. THE WAVEFORM PARAMETER OF KANGSHIFU PURE WATER 
Among the liquid drop fingerprint extraction methods, waveform analysis method is simple, effective and also widely applied. Figure 3 are the liquid drop fingerprint with the aforementioned method, and the abscissa and ordinate correspond respectively to the capacitance signals and optical signals, which are the voltage data obtained from the A/D board through the signal processing circuit. In this paper, they are referred to as capacitor voltage signals and fiber voltage signals.

These 10 characteristic values (10-dimensional vector) contain the main features of each liquid drop fingerprint and these values can be considered to represent a kind of liquid. After further pattern recognition to these 10 eigenvalues, the liquid can be identified [1,3].

\section{THE OPTIMIZATION ON ABNORMAL VALUES OF THE LIQUID DROP FINGERPRINT}

\section{A. The Detection of Abnormal Droplet}

In the process of the formation of droplets, the droplets are inevitably affected by many factors, such as: air bubbles or impurity in the droplets, recirculation system jitter, uneven illumination and so on. The fiber capacitance signals of droplets in these interference circumstances are very different with them generated under normal conditions. The fiber capacitance signals with abnormal drops of grape wine Shuangkou are showed in Figure 3.

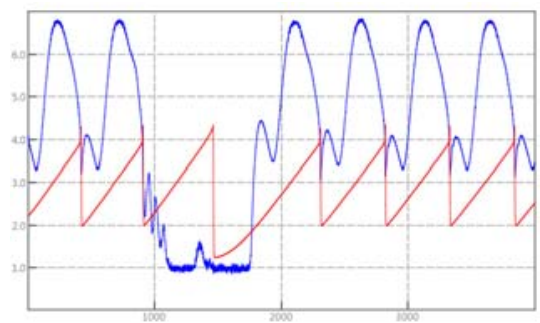

FIGURE III. THE FIBER CAPACITANCE SIGNALS WITH ABNORMAL DROPS OF GRAPE WINE - SHUANGKOU

Removing the first incomplete droplet and counting the next droplet as the first one, there are obvious differences in the optical fiber voltage values between the second droplet and normal droplets. The capacitor voltage values of the third droplet also have big difference with the normal. Thus the second and the third droplets can be considered as abnormal droplets.

Abnormal droplets can bring tremendous harm. Because the liquid drop fingerprint is derived by the normalization of all droplets, and the formation of the abnormal droplet will result in inaccurate liquid drop fingerprint, which can achieve erroneous results in pattern recognition. Thus, detecting and removing the abnormal liquid droplet is an important aspect.

Factors in the formation of abnormal droplets are various, and it is difficult to completely take into account them in the droplet generation process. So it is unrealistic to avoid the formation of abnormal droplet.

\section{B. Feature Data of Droplets with Feature Extraction}

Each individual droplet fiber voltage signals and the capacitor voltage signals can be considered as a droplet fingerprint. The droplet fingerprint feature extraction method can be used to extract droplets feature data. The waveform analysis method is used to optical fiber voltage signals for feature extraction, and the linear fitting method is used to capacitor voltage signals for feature extraction. Therefore, each droplet can be extracted to 12-dimensional feature vector, to grape wine - Shuangkou for example, the droplet eigenvalues data in Table 1.

TABLE I. THE DROPLETS FEATURE DATA OF GRAPE WINE SHUANGKOU OBTAINED BY FEATURE EXTRACTION

\begin{tabular}{|c|c|c|c|}
\hline $\begin{array}{l}\text { number } \\
\text { feature } \\
\text { values }\end{array}$ & 1st & 2nd & 3rd \\
\hline P1 & 452 & 949 & 1466 \\
\hline P2 & 31 & 12 & 3 \\
\hline P3 & 115 & 26 & 8 \\
\hline P4 & 296 & 37 & 11 \\
\hline P5 & 487 & 483 & 835 \\
\hline P6 & 3.23124 & 2.32311 & 1.3243 \\
\hline P7 & 0.71234 & 0.87234 & 0.0242 \\
\hline P8 & 3.53412 & 0.53123 & 0.0412 \\
\hline P9 & 1922.43 & 9656.21 & 6342.32 \\
\hline P10 & 51.21 & 25.48 & 54.23 \\
\hline P11 & 2.92122 & 2.87231 & 2.86223 \\
\hline P12 & 0.56518 & 0.58453 & 0.23512 \\
\hline $\begin{array}{l}\text { feature } \\
\text { values }\end{array}$ & 4th & 5th & 6th \\
\hline P1 & 2315 & 2829 & 3321 \\
\hline P2 & 34 & 32 & 36 \\
\hline P3 & 119 & 121 & 113 \\
\hline P4 & 289 & 301 & 287 \\
\hline P5 & 488 & 494 & 481 \\
\hline P6 & 3.31341 & 3.29881 & 3.28691 \\
\hline P7 & 0.73213 & 0.69872 & 0.74534 \\
\hline P8 & 3.67451 & 3.51231 & 3.62411 \\
\hline P9 & 1992.98 & 1966.71 & 1988.04 \\
\hline P10 & 51.65 & 51.45 & 52.66 \\
\hline P11 & 2.97434 & 2.97158 & 2.89761 \\
\hline P12 & 0.55234 & 0.55701 & 0.58987 \\
\hline
\end{tabular}

The feature data of all liquid droplets can be great extracted by using waveform analysis method and capacitance linear fitting method. However, because of large number and uncertain quantity of droplets, this method requires a higher standard of procedures, so a more easy way to extract droplet characteristics data should be adopted to solve the relatively high dimension of eigenvalues and the high time complexity of the algorithm. 


\section{Optimization on Feature Extraction Method of Droplet Data}

The purpose of extracting characteristic data of the droplet is not to recognize it, but to identify abnormal droplets. Therefore, the feature extraction method is not very complex, which can only extract some representative data.

Figure 3 shows that both the fiber voltage signals and the capacitor voltage signals of abnormal drops can be likely unusual. Therefore, this paper extracted droplet time sequence length, the starting value of fiber voltage, the ending value of fiber voltage, the initial value of capacitor voltage, the termination value of the capacitor voltage and the maximum value of fiber voltage to form characteristic data, which can compose a new six-dimensional droplet feature data of grape wine - Shuangkou. The results are obtained as shown in Table 2.

TABLE II. THE OPTIMIZED DROPLETS FEATURE DATA OF GRAPE WINE - SHUANGKOU

\begin{tabular}{|c|l|l|l|}
\hline $\begin{array}{c}\text { number } \\
\text { feature } \\
\text { values }\end{array}$ & \multicolumn{1}{|c|}{ 1st } & \multicolumn{1}{|c|}{ 2nd } & 3rd \\
\hline P1 & 487 & 483 & 835 \\
\hline P2 & 3.55167 & 2.45288 & 0.98064 \\
\hline P3 & 3.11966 & 1.05766 & 3.60544 \\
\hline P4 & 1.97065 & 1.99360 & 1.22864 \\
\hline P5 & 3.00429 & 1.62817 & 2.98022 \\
\hline P6 & 6.76342 & 3.19873 & 6.81221 \\
\hline \multicolumn{4}{|l}{} \\
\hline $\begin{array}{l}\text { number } \\
\text { feature } \\
\text { values }\end{array}$ & 4th & $\mathbf{5 t h}$ & 6th \\
\hline P1 & 488 & 494 & 481 \\
\hline P2 & 3.64550 & 3.54375 & 3.55068 \\
\hline P3 & 3.47666 & 3.46596 & 3.46523 \\
\hline P4 & 1.98168 & 1.98183 & 1.98437 \\
\hline P5 & 2.97983 & 2.98083 & 2.97900 \\
\hline P6 & 6.75213 & 6.71235 & 6.83421 \\
\hline
\end{tabular}

\section{THE BoXplot Method AppliEd to the Detection OF THE LIQUID DROP FINGERPRINT'S ABNORMAL VALUES}

Boxplot is used to display a chart of the dispersion of a set of data, which can be seen whether data has the symmetry of information distribution and the degree of dispersion, so directly and clearly identify the data outliers [4].

According to the box plots of the meaning and with the characteristics, which can be used to detect abnormal droplet. Draw all the droplets of the same dimensional data, observe whether there is abnormal data, and record the number corresponding the abnormal droplet. Draw the boxplot with 6-dimensional data of all droplets. As long as a feature value is abnormal, the droplet can be identified as abnormal droplets.

According to the boxplot method of drawing out 6 dimensional droplet characteristic data of grape wine Shuangkou, which are shown in Figure 4.
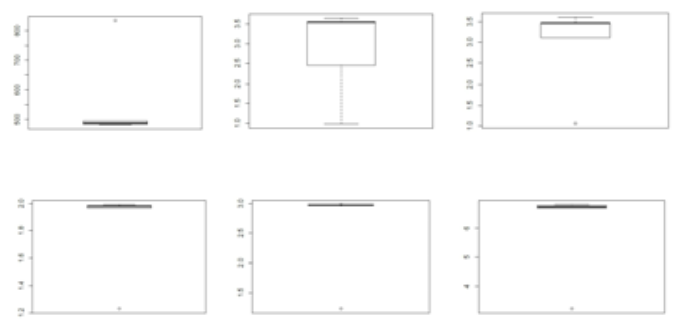

FIGURE IV. THE BOXPLOT WITH SIX-DIMENSIONAL FEATURE DATA OF GRAPE WINE - SHUANGKOU

The Table 3 can be obtained in above 6 boxplot, and the $\mathrm{X}$ indicates that the dimension data of the droplets is abnormal. No mark says that the dimension data of the droplets is normal.

TABLE III. THE ABNORMAL DROPLET DATA STATISTICS OF GRAPE WINE - SHUANGKOU

\begin{tabular}{|c|l|l|l|l|l|l|}
\hline $\begin{array}{c}\text { number } \\
\text { feature } \\
\text { values }\end{array}$ & 1st & 2nd & 3rd & 4th & 5th & 6th \\
\hline P1 & & & $\mathrm{X}$ & & & \\
\hline P2 & & & & & & \\
\hline P3 & & $\mathrm{X}$ & & & & \\
\hline P4 & & & $\mathrm{X}$ & & & \\
\hline P5 & & $\mathrm{X}$ & & & & \\
\hline P6 & & $\mathrm{X}$ & & & & \\
\hline
\end{tabular}

According to the Table 3, the 2nd and the 3rd liquid drop of grape wine - Shuangkou are abnormal, which is coinciding with the actual situation.

In abnormal drop detection, when one data is determined to be normal, the data may be combined with a certain floating value (5\% to $10 \%$ ) as a threshold value. Accordingly, it can be determined whether all the rest of the data is normal. If all data will be performed the same operation with this method, all drops be can determined, and the purpose of detecting abnormal droplets is achieved.

Boxplot shows the median number of data. When the number of normal droplets is much than the number of abnormal droplets, the fluid can be considered to be successful. So the median data for each dimension are inevitable to be normal data. With the median $( \pm 5 \%)$ as the threshold values, abnormal droplets are screened.

\section{CONCLUSION}

The process of droplet generation is inevitably affected by many factors, droplets fiber capacitance signals in these interference situation is very different from those formed normally. The impact of these disturbances on the droplet analysis system first appeared on the original data, resulting in abnormal droplets data. Without these exceptions data 
processing, it will make the droplets fingerprint abnormal, leading to system instability, and thus will affect the recognition accuracy of the droplet analyze system.

According to the characteristics of abnormal liquid drop fingerprint and the mentality of waveform analysis method on feature extraction of the liquid drop fingerprint, the functional optimization is applied in the 12 dimensional feature vectors of liquid drop. The droplet time sequence length, the starting value of fiber voltage, the ending value of fiber voltage, the initial value of capacitor voltage, the termination value of the capacitor voltage and the maximum value of fiber voltage are extracted to form characteristic data. Optimizing the 12 dimensional feature vectors of the liquid drop fingerprint and then using boxplot method on the optimized 6 dimensional feature vectors for visualization of statistics. Based on median $( \pm 5 \%)$ threshold, the abnormal droplets are screened. Experimental results show that the detection recognition ratio of the abnormal liquid drop can be ensured after feature optimization, and the time complexity of the algorithm is greatly reduced. Boxplot method is effective in detection of abnormal liquid drop fingerprint, with its accuracy up to $100 \%$ among selected samples.

\section{ACKNOWLEDGMENT}

Especial thanks to The Natural Science Foundation of China (NSFC) and Beijing Higher Education Young Elite Teacher Project for the financial support.

\section{REFERENCES}

[1] Q. Song, Research of the identification method based on Drop Analysis Technology and Droplet Fingerprint. Tianjin University. 2005.

[2] Y. Luo, Q. Song, J. Li, C.W. Zou, Fuzzy pattern recognition method applied in droplet fingerprint recognition [C], Computer Application and System Modeling, 2010 International Conference on. IEEE, 2010.

[3] Z.R. Qiu, X.H. Chen, X.H. Li, Fiber capacitance drop analyzer and its recognition algorithm, Journal of Tianjin University. 44(5). 445449.2011.

[4] Y. Xue, L.P. Chen, Statistical Modelin And R Language. Tsinghua University Press. 2007.4 